\title{
Generation of dark, Q-switched and bright pulses using erbium-ytterbium-thulium co- doped fiber
}

\begin{abstract}
We demonstrate the generation of dark and bright pulses in a Q-switched laser using an erbiumytterbium-thulium co-doped fiber. By manipulating pump power while maintaining lasing wavelength at $1571 \mathrm{~nm}$, dark and bright pulses were observed at pump power thresholds 94.1 and $1770 \mathrm{~mW}$ with pulse duration of 3 and $71 \mathrm{~ns}$, respectively. Stability of the two regimes were also high across an operating time of $60 \mathrm{~min}$. These findings may be the stepping stone towards realising a laser with flexible operating range at different pulse regimes.
\end{abstract}

Keyword: Dark pulses; Q-switching; Bright pulses; Erbium-doped fiber laser 